\title{
Virtual reality exergaming as adjunctive therapy in a sub-acute stroke rehabilitation setting: facilitators and barriers
}

Ai-Vi Nguyen, Yau-Lok Austin Ong, Cindy Xin Luo, Thiviya Thuraisingam, Michael Rubino, Mindy F. Levin, Franceen Kaizer \& Philippe S. Archambault

To cite this article: Ai-Vi Nguyen, Yau-Lok Austin Ong, Cindy Xin Luo, Thiviya Thuraisingam, Michael Rubino, Mindy F. Levin, Franceen Kaizer \& Philippe S. Archambault (2019) Virtual reality exergaming as adjunctive therapy in a sub-acute stroke rehabilitation setting: facilitators and barriers, Disability and Rehabilitation: Assistive Technology, 14:4, 317-324, DOI:

10.1080/17483107.2018.1447608

To link to this article: https://doi.org/10.1080/17483107.2018.1447608

Published online: 12 Mar 2018.

Article views: 835

View Crossmark data \lceil
Submit your article to this journal 주

View related articles $\asymp$

Citing articles: 9 View citing articles 


\title{
Virtual reality exergaming as adjunctive therapy in a sub-acute stroke rehabilitation setting: facilitators and barriers
}

\author{
Ai-Vi Nguyen ${ }^{a}$, Yau-Lok Austin Ong ${ }^{a}$, Cindy Xin Luo ${ }^{a}$, Thiviya Thuraisingamª ${ }^{a}$ Michael Rubino ${ }^{a}$, Mindy F. Levin ${ }^{a, b}$, \\ Franceen Kaizer $^{\mathrm{b}, \mathrm{c}}$ and Philippe S. Archambault ${ }^{\mathrm{a}, \mathrm{b}}$

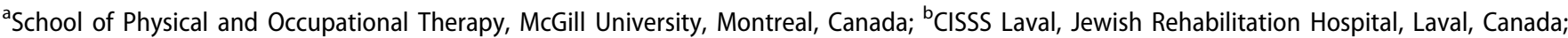 \\ Interdisciplinary Research Centre in Rehabilitation (CRIR), Montreal, Canada
}

\begin{abstract}
Purpose: To identify the facilitators and barriers perceived by clinicians to using an Exergaming Room as adjunct to conventional therapy.

Design: Phenomenological qualitative study using an interpretive description methodology.

Subjects: Ten clinicians (four physical therapists, six occupational therapists) from the Stroke Program at the Jewish Rehabilitation Hospital (nine female, one male, age range 25-50 years old) who referred clients to the Exergaming Room.

Methods: Ten to twenty minute semi-structured interviews were conducted with each clinician. Convenience sampling was used. A thematic analysis was performed on the data collected by grouping all the open codes into facilitators and barriers, and then categorized into levels, themes and subthemes.

Results: Facilitators and barriers were divided into three levels: organizational, individual and technological. Major facilitators at the organizational level were: institutional support; at the individual level: personal experience of referring clinician, presence of an expert clinician, and relevance of the Exergaming Room for stroke clients; and at the technological level: perceived ease of use of the exergames and possibility of providing additional therapy. Key barriers to successful implementation of the Exergaming Room at the organizational level were: scheduling difficulties and lack of staffing; at the individual level: client functional limitations; at the technological level: low precision in motion capture of the exergame systems.

Conclusions: Multiple factors affect the implementation of new technology in rehabilitation settings. In order to successfully integrate exergame systems into practice, institutions are encouraged to take the identified factors (facilitators and barriers) into account.
\end{abstract}

\section{ARTICLE HISTORY}

Received 25 September 2017 Revised 23 February 2018 Accepted 27 February 2018

\section{KEYWORDS}

Stroke; rehabilitation; exergames; serious games; qualitative methodology
- IMPLICATIONS FOR REHABILITATION

- Clinicians who have referred individuals with stroke to an "exergames" room over a 1-year period at a rehabilitation hospital have found the service to be highly relevant to their clients.

- The presence of an expert clinician, who evaluates the clients and builds an exergames activity program, was seen as an important facilitator by referring clinicians in the use of this service.

- An ideal Exergames Room should offer a wide variety of activities, including some that focus on motor, cognitive and/or communications abilities.

\section{Introduction}

In recent years, virtual reality (VR) commercial consoles, also known as exergaming, have emerged as a new form of intervention for stroke rehabilitation. Such systems allow individuals to participate in tasks ranging from exercises to the completion of purposeful activities within a virtual environment. VR is defined as "interactive simulations created with computer hardware and software to present users with opportunities to engage in environments that appear and feel similar to real world objects and events" [1]. Multiple studies have reported significant improvements in functional outcome measures when clinicians use exergames as adjunctive therapy in stroke rehabilitation [2-5]. For instance, a systematic review by Laver et al. reported significant improvement in upper limb (UL) function of individuals with stroke when using VR compared to alternative intervention methods or to the absence of an intervention [3]. In addition, when compared to repetitive exercises, the practice of purposeful movements elicits a greater quantity and quality of UL movements, making it an integral part of promoting motor system connectivity, and improving functional status post-stroke [6-8].

Clinicians generally express positive feelings about using VR for the treatment of individuals with stroke. According to Levac and Miller [9], the main facilitators identified by clinicians for using VR in clinical practice include its ability to provide multisensory feedback, mimic real-life situations, and increase client motivation to allow for increased practice intensity. On the other hand, barriers influencing the implementation of VR in clinical settings have also been described and include a lack of time and knowledge to gain familiarity with the technology [10-12], distraction from rehabilitation goals, and limited options to individualize treatment plans 
through game options [11]. Furthermore, the implementation of a new technology for assisting UL recovery in stroke has also been examined. Hochstenbach-Waelen and Seelen [13] concluded that for a technology to be well received by therapists, therapist perspectives on the usability and usefulness of the technology are crucial factors. Key features provided by the technology important to clinicians were the opportunity to engage in goal-oriented therapy, the considerations of cognitive impairments, the resemblance to real-life contexts, the possibility of varying the type of exercise and adapting exercise difficulty through increasing intensity and frequency of task-related movements, the opportunity for feedback, as well as the motivation and challenge for clients [13].

To leverage the potential benefits of VR for the rehabilitation of individuals with stroke, an "Exergames Room" was set-up at the Jewish Rehabilitation Hospital of the Centre Intégré de Santé et de Services Sociaux de Laval (JRH, Laval, Canada) in March 2015, while considering the barriers and facilitators already identified in the literature as mentioned in the previous paragraphs. Specifically, two different VR systems were carefully selected to offer a variety of exercises and activities, with a wide range of difficulty levels: the Meditouch and the Jintronix Wave. To address the issue of familiarity with technology, clinicians could refer individuals with stroke to an expert clinician, who had been specifically trained on each of the systems and who would then create an exergames program based on client goals identified by referring clinicians.

The overall aim of this study was to describe the experiences of clinicians who had referred individuals with stroke to the Exergaming Room, one year after program implementation. This knowledge will serve to identify barriers and facilitators to the implementation of an exergaming service specifically tailored to a stroke rehabilitation patient population. This independent study did not involve the participation of patients.

\section{Methods}

An interpretivist research paradigm was used in this qualitative study to gain a deeper understanding of how clinicians working in a Stroke Program incorporate the use of VR in their professional practice. This paradigm seeks to understand their viewpoint based on their reality surrounding a particular topic [14]. More precisely, the interpretive description was used to explore clinicians' attitude towards VR and explain the factors promoting, and those restricting the use of VR.

\section{Participants}

To participate in the research project, interviewees needed to have practiced as a physical or occupational therapist in the Stroke Program of the JRH either part-time or full-time. Clinicians were excluded if they were unable to participate in a live interview. A detailed description of the purpose and methodology of the study was provided to clinicians during interdisciplinary rounds. Participants signed informed consent forms approved by the Ethics Committee of the Centre for Interdisciplinary Research in Rehabilitation (CRIR). Ten clinicians (92\% female) from the Stroke Program of the JRH were recruited for interviews, including six occupational therapists (six female) and four physical therapists (one male, three female) using convenience sampling with a mean age of 34.5 years old. Nine out of 10 participants had worked in the Stroke Program since the implementation of the Exergaming room. All participants had referred clients to the Exergames Room over the past year. Client referral was based on clinical judgement of potential therapeutic benefits.

\section{Exergames room}

The Exergames Room was established at the JRH in March 2015. Approximately, 9 months were required to obtain the room and approval from the hospital administration, to purchase the equipment, to establish the referral process and to hire the personnel. The Exergames Room contains two systems where clients could practice outside of their regular therapy sessions. The Jintronix is an example of a modern VR rehabilitation software compatible with the Xbox Kinect. It combines markerless motion capture technology with clinically designed games to elicit purposeful movements that can be done in sitting or standing [15]. The Meditouch HandTutor consists of a set of electrogoniometers measuring wrist and elbow movements and interfaced with a VR rehabilitation software system. It allows the repetition of functional UL movements within a game context, while providing augmented motion biofeedback [16].

The Exergames Room staff included an expert clinician, who was present one half-day per week, and an assistant, who was present an additional two to three half-days per week. Physical or occupational therapists in the Stroke Program could refer clients to the Exergames Room by completing a referral form, in which two options were available. The first one was for the therapist to specify overall treatment goals, such as improving UL range of motion, sitting balance, endurance, etc. Then, the expert clinician, who was a certified occupational therapist would select the appropriate exergame system and activities during an initial evaluation session, according to the stated goals. Following the evaluation, the assistant would work with the client on a weekly basis to complete the exergame program established by the expert clinician. The second option was for the therapist to choose the exergame activities themselves, with or without the help of the expert clinician. In subsequent sessions, the assistant would be responsible for setting up the exergame systems and for supervising the clients' participation in the activities. Therapists chose their preferred option depending on their level of familiarity with the technology. For either option, the Exergame Room assistant established a weekly schedule based on the client's availabilities.

\section{Data collection}

Participants completed a short demographic questionnaire online and then took part in an interview lasting approximately 10-20 min. The interviews took place in a closed and quiet room to insure confidentiality. Two researchers interviewed each clinician in their preferred language, either English or French. A lunch voucher and tea with cookies were offered as compensation for participants' time (Table 1).

A list of open-ended questions was developed based on a technology acceptance model: the Unified Theory of Acceptance

Table 1. Demographic data of participants.

\begin{tabular}{ccccc}
\hline Clinician \# & $\begin{array}{c}\text { Profession } \\
\text { (OT/PT) }\end{array}$ & $\begin{array}{c}\text { Experience in } \\
\text { neurology (years) }\end{array}$ & Age (years) & Sex (M/F) \\
\hline 1 & OT & 2 & 25 & $\mathrm{~F}$ \\
2 & OT & 2 & 31 & $\mathrm{~F}$ \\
3 & OT & 10 & 32 & $\mathrm{~F}$ \\
4 & PT & 10 & 35 & $\mathrm{~F}$ \\
5 & PT & 25 & 43 & $\mathrm{~F}$ \\
6 & PT & 9 & 50 & $\mathrm{~F}$ \\
7 & PT & 9 & 40 & $\mathrm{M}$ \\
8 & PT & 6 & 32 & $\mathrm{~F}$ \\
9 & OT & 11 & 34 & $\mathrm{~F}$ \\
10 & OT & 1 & 34 & $\mathrm{~F}$ \\
11 & OT & 6 & 27 & $\mathrm{~F}$ \\
12 & OT & & 31 & $\mathrm{~F}$ \\
\hline
\end{tabular}


Table 2. Theme definition.

Virtual reality in sub-acute stroke setting

Organizational level

Communication

Referral process

Scheduling process

Training process

Reinforcement of clinician's use

Staffing process

Environmental

Resources

Length of session

Individual level

Referring clinician factors

Knowledge of VR

Clinicians' personal experience

Positive outlook of VR

Trial sessions

Personal factors

Expert clinician factors

Communication

Client safety

Practicality

Reliability

Client factors
Definition

Factors pertaining to the use of VR in a sub-acute stroke rehabilitation setting including organizational factors, individual factors and technology factors.

All Jewish Rehabilitation Hospital (JRH)'s operations put in place to facilitate the use of the Exergaming Room including logistical processes related to the use of the Exergaming Room. Weaknesses in the JRH's processes related to the Exergaming Room including barriers in the domains of communication, referral, training, scheduling and staffing.

Verbal and non-verbal exchanges between healthcare professionals regarding referral process and use of room including team decisions and discussions, and social influences. Lack of feedback between healthcare providers including comments on lack of verbal and nonverbal feedback.

Clinicians' perspective on the how to register client to Exergaming Room including method of referral and clinicians' attitudes towards the referral process.

Complications or weaknesses related to the method of registering a client to the Exergaming Room including limitations of the referral form.

Feasibility of client arrangement to Exergaming Room including availabilities, accessibility of the schedule, and clinician opinion on scheduling.

Dilemmas relating to appointing time slots to client for the Exergaming Room including lack of schedule availabilities and lack of access to schedule.

Educational sessions provided by the institution on Jintronix, MediTouch and the referral process Including different types of training and information mediums. Issues pertaining to the process of gaining knowledge and experience with the Exergaming Room including barriers related to length, frequency, and quality of training.

Actions and players within the facility encouraging referral to Exergames Room including reminders, encouragements and key people.

Lack of trained personnel to supervise the Exergaming Room including lack of supervision and staff.

Physical and temporal resources available at the JRH including place and material. Limitations in the JRH in terms of means available including lacks in material and temporal resources.

Available resources required for the function on the Exergames Room. Lack of material contributing to lower usage of Exergaming Room including insufficient space and material.

Lack of time spent in the Exergaming Room adjunctive therapy session including insufficient time.

Facilitators related to different individuals in a stroke rehabilitation setting in the JRH including factors pertaining to main actors (client, referring clinicians and expert clinician). Factors which impede the use of the Exergaming Room at the level of the users of the room including factors related to different key actors involved in the use of the Exergaming Room.

Positive factors that influences clinicians' decision to refer clients to the Exergaming Room including clinicians' attitudes, experience, and perceptions about the room.

Clinician-related factors which prevent or impede their referral decision including clinician's motivation, insufficient personal experience, and perceived risk for patients.

Position towards best practice guidelines influencing their decision to refer to the Exergaming Room including attitudes towards best practice guidelines. Lack of knowledge on VR hardware and software operations including clinicians' lack of knowledge on how Jintronix and Meditouch works.

Influence of clinicians' past experiences with video game therapy and VR on their decision to refer clients to the Exergaming Room including focus groups and hands-on experience with VR on-site.

Positive attitudes clinicians have adopted towards VR and its future including beliefs about the future of the Exergaming Room.

Opportunity given to clients by clinicians to try out the Exergaming Room including attitudes towards trial sessions.

Clinician's individual lived experiences impeding their decision to refer to the Exergaming Room including lack of experience, exposure, and familiarity with VR.

Positive factors as perceived by others related to the Exergaming Room's expert clinician including communication between expert clinician and referring clinicians, room supervision, practicality, and reliability factors related to the expert clinician role. Negative factors as perceived by others related to the expert clinician including interactions between referring clinician and expert clinician.

Clinicians' positive feedback about information exchange between expert clinician and themselves including quantity and quality of communication exchanged. Clinicians' negative feedback about information exchange between expert clinician and themselves including lack of verbal and non-verbal exchange between the implicated parties.

Ways in which the expert clinician ensures a safe environment within the Exergaming Room for the clients including actions taken prior to and during sessions.

Advantages of having an expert clinician manage the Exergaming Room including ways in which expert clinician facilitates referring clinicians' use of the Exergaming Room.

The trust that referring clinicians have towards the expert clinician including expressions of trust and confidence.

Client related variables reinforcing the use and referral to the Exergaming Room including applicability factors, motivational factors and positive client feedback. 
Table 2. Continued

\begin{tabular}{|c|c|}
\hline Theme & Definition \\
\hline & $\begin{array}{l}\text { Client related factors which prevent or impedes the clinicians' referral decision including personal } \\
\text { factors and client functional limitations. }\end{array}$ \\
\hline \multirow[t]{2}{*}{ Personal factors } & $\begin{array}{l}\text { Client characteristics/features that impede clinicians' referral decision including age, personal interest, } \\
\text { finance level and confidence level. }\end{array}$ \\
\hline & $\begin{array}{l}\text { Client characteristics/features which impede clinicians' referral decision including age, personal } \\
\text { interest, finance level and confidence level. }\end{array}$ \\
\hline Applicability to different levels of functions & $\begin{array}{l}\text { Variety of client that may benefit from using the Exergaming Room including tolerance to activity, } \\
\text { independence level and endurance level. }\end{array}$ \\
\hline Feedback from clients & $\begin{array}{l}\text { Positive responses clients have given to the referring clinicians via feedback about the Exergaming } \\
\text { Room including positive feedback from clients. }\end{array}$ \\
\hline Functional limitations & $\begin{array}{l}\text { Restrictions in clients' level of function which negatively influences their participation to the } \\
\text { Exergaming room including description of clients' disability. }\end{array}$ \\
\hline Technological level & $\begin{array}{l}\text { The direct facilitators found with the use of the Jintronix and Meditouch in the Exergaming Room } \\
\text { for a stroke rehabilitation clientele including gaming system features and its benefits. } \\
\text { The direct barriers found with the use of the Jintronix and Meditouch in the Exergaming Room } \\
\text { for a stroke rehabilitation clientele including gaming system features and its barriers. }\end{array}$ \\
\hline VR system & $\begin{array}{l}\text { The Jintronix and the Meditouch system along with the games offered including diversity games/ } \\
\text { goals, ease of use, system feedback. } \\
\text { The limitations Jintronix and the Meditouch systems along with the games offered including } \\
\text { weaknesses in VR system and games available. }\end{array}$ \\
\hline Games & $\begin{array}{l}\text { The range of games the VR system offers considering clients' functional abilities and difficulty levels } \\
\text { including different range of games and adjustable difficulty level. } \\
\text { Choice of games that are lacking in the VR systems (Jintronix and Meditouch) including lack of } \\
\text { game choices for different functional goals and levels. }\end{array}$ \\
\hline Clients'/clinicians' perceived ease of use & $\begin{array}{l}\text { The degree to which a person believes that using a particular system would be free from effort [22] } \\
\text { including beliefs on the ease of use of the Jintronix and Meditouch. }\end{array}$ \\
\hline VR system feedback & $\begin{array}{l}\text { Indicators of client performance on tasks displayed by the VR system in order for clients to improve } \\
\text { including scores and movement feedback. }\end{array}$ \\
\hline Performance of VR system & $\begin{array}{l}\text { Innate flaws of VR in a rehabilitation setting include motion sensor flaws, complexity of software, } \\
\text { and limited ability to simulate real world tasks. }\end{array}$ \\
\hline Therapeutic benefits & $\begin{array}{l}\text { Advantages of the Exergaming Room as an adjunct therapy in a stroke rehabilitation setting include } \\
\text { supplemental rehabilitation options and rehabilitation benefits. }\end{array}$ \\
\hline Additional therapy & $\begin{array}{l}\text { Exergaming Room as supplemental rehabilitation given outside of standard physiotherapy or } \\
\text { occupational therapy sessions including extra therapy time and more movement repetitions. }\end{array}$ \\
\hline Rehabilitation benefits & $\begin{array}{l}\text { Potential therapeutic objectives for clients attending the Exergaming Room sessions including variety } \\
\text { of potential goals including functional and spiritual benefits. }\end{array}$ \\
\hline
\end{tabular}

and Use of Technology (UTAUT) [17]. The UTAUT is a model designed to predict the likelihood of a technology being accepted by its users based on four constructs: performance expectancy, effort expectancy, social influence and facilitating conditions. In addition, the list included open-ended questions to get participants' general impression of the Exergaming Room. The interview questions were pilot tested within the team of five researchers, to ensure clarity. In addition, the semi-structured interview format allowed for participants to openly share their experience. Examples of questions included: "What would facilitate or impede your willingness to refer a client to use VR as adjunctive therapy?" and "How do you find the referral process?" Each interview was conducted by two researchers collaboratively, with the aim of maximizing information collected using probing questions. For the same purpose of gathering a greater diversity of responses, each pair of interviewers were formed by different members of the team. The interviews were audio recorded and transcribed verbatim by the same pair of researchers who had conducted the interview. A code was assigned to each participant allowing for anonymization of the data.

\section{Data analysis}

The first layer of coding consisted of reading the transcripts individually to become familiar with the data. The open coding was completed individually since opinions on the importance of quotes could have varied. As a result, additional codes were identified. In order to triangulate findings, two researchers open-coded each interview in English, regardless of the original language of the interview, by pen and paper analysis. These two were different than the ones who had initially performed the interview in order to minimize carry-over bias (i.e., in the case that researchers 1 and 2 conducted a particular interview, researchers 3 and 4 could then code the data). After this first layer of coding, the resulting findings were then synthesized (e.g., combining codes). Codes were first separated into facilitators and barriers; then a thematic analysis with a bottom-up approach was conducted [18]. Concepts were collaboratively identified and defined from the open codes by all evaluators. Each level, theme and subtheme were defined and each code was verified to ensure proper allocation (Table 2). The themes were refined collaboratively throughout the study as further insight was gained to generate an optimal representation.

\section{Results}

Figure 1 depicts the different themes and levels (organizational, individual and technological) of barriers and facilitators as perceived by clinicians. The figure was inspired by the Organizational Behaviour Framework [19]. This allowed for a greater understanding of factors that influenced clinicians' behaviours to refer clients to the Exergames Room within the organizational setting. This framework was originally divided into three levels: organizational, group and individual. However, the "group" level was omitted as the interaction between individuals was categorized under "individual". A "technological" level was added to incorporate the influence of VR system-related factors on clinicians' perceptions. Only the most significant themes are presented. The latter was 


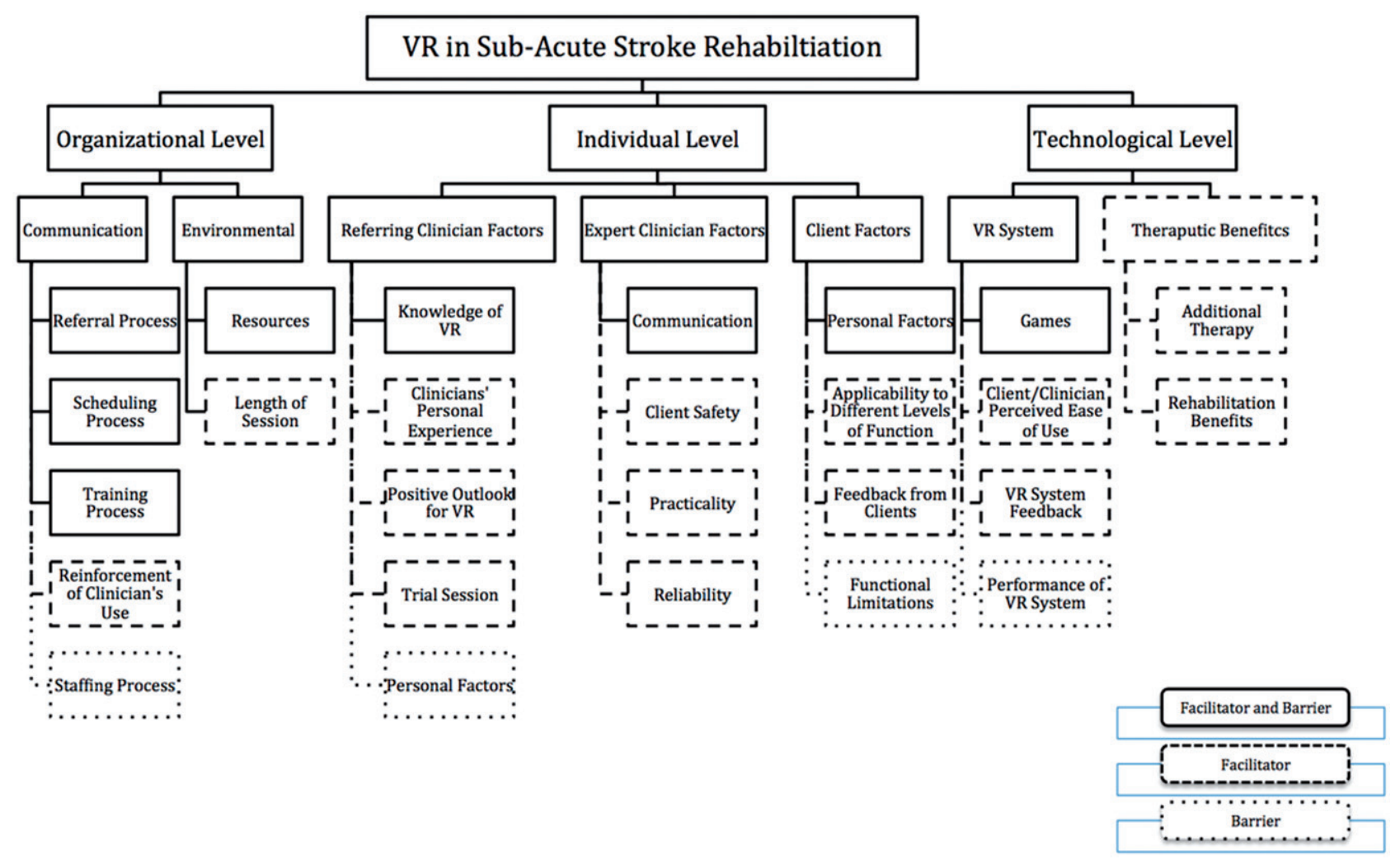

Figure 1. Thematic tree of facilitators and barriers to using virtual reality (VR) exergames in a stroke rehabilitation setting.

determined based on "repetition across participants" and "amount of elaboration/details".

\section{Organizational level}

\section{Institutional}

Communication (facilitators and barriers). Clinicians mentioned that team discussions during interdisciplinary rounds helped facilitate the referral of clients to the Exergames Room. As well, there were social influences between clinicians which caused "[translated] a domino effect", as one clinician described. However, some clinicians noticed the lack of discourse on the Exergaming Room and its use.

Referral process (facilitators and barriers). Most clinicians expressed their satisfaction with the referral process. They found the referral form to be easy and efficient. As one participant described, clinicians only had to "write the objectives and let [the expert OT] handle the rest". Only one clinician reported that use of the referral form was time-consuming. Another clinician suggested incorporating cognitive objectives on the referral form since current options only included physical components such as sitting and standing balance, upper and lower limb practice, as well as endurance training.

Scheduling process (facilitators and barriers). Since the implementation of the Exergames Room, additional time slots were added, increasing the availability of the room and thus facilitating referrals. Moreover, therapists felt that they had an easier time referring in-patients compared to out-patients due to the logistics involved with other groups and timing. Another barrier was the lack of access to the room schedule for clinicians, which complicated schedule modifications. Therapists working in the inpatient unit did not have easy access to the hospital's computer scheduling system, as they used a magnetic board to indicate their daily schedules. This led to a lack of flexibility when rescheduling patients for other therapy sessions was necessary.
Training process (facilitators and barriers). As a facilitator, clinicians reported that receiving training for the Exergames Room added to their ability to properly refer clients. However, therapists admitted that "the training [the institution] gave [them was] really limited and [...] too general". The amount of training was perceived as insufficient due to a lack of hands-on practice with the VR systems themselves.

Reinforcement of clinician's use (facilitators). Many clinicians mentioned they were reminded to use the Exergames Room through emails, rounds and other means. Also, they felt like the link between the research and clinical departments acted as a facilitator. One clinician mentioned that "[the room] is very encouraged here [...] it's a facilitator that the institution supports best practice and supports wanting to give the best for the clients".

Staffing (barriers). Most clinicians found the lack of staff and supervision in the room to be a barrier. In other words, this implied that there were not enough time slots available to use the room throughout the week. One clinician suggested that the wait time for clients was a consequence of a lack of staff to manage the room. Another participant suggested turning the Exergames Room into a group therapy space for outpatients to allow more clients to participate at the same time, making it more time-efficient.

\section{Environmental}

Resources (facilitators and barriers). The accessibility of the room to patients was identified as a facilitator in addition to the small amount of resources required to operate the room. It was mentioned that the setup was optimal for the available space. However, some barriers were identified such as the need for more varied exergames systems, additional rooms and more space. One clinician suggested the addition of a plinth in the room to facilitate UL movement as wheelchair armrests may hinder range of motion when performing some of the games. Others mentioned the need for more cleaning supplies to help with infection control. 
Length of session (barriers). Clinicians mentioned the length of session to be a barrier as it was generally too short. As one clinician reported: "My patients told me that they came all the way downstairs and it's only 10 to $15 \mathrm{~min}$ [...] whereas the GRASP [a hand activity program] is longer periods of time so they feel that even though it's once a week, they feel that it's more worth it because of the fact that it's longer."

\section{Individual level}

\section{Referring clinician factors}

Knowledge of VR (facilitators and barriers). Some clinicians stated that they were familiar with the benefits of VR, and that they were aware of the evidence of VR for increasing movement repetitions, which was seen as a facilitator. However, at least three clinicians were not familiar with how the VR systems actually worked and how to operate the games. This acted as a barrier for clinicians to use the room with their clients.

Clinician's personal experiences (facilitators). Half of the clinicians linked their experience with exergames as a positive influence towards their referral decision. Their motivation to refer stemmed from their familiarity with the systems. This allowed them to experience the benefits first-hand and understand the purpose of the exergaming program. One clinician stated: "[translated] Personally, since I have experience in the private clinic using modalities like video games [...] I have seen benefits with my patients so, in terms of experience, this incited me to think that the Exergaming Room was a good idea." Finally, clinicians reported feeling more capable of making accurate referral decisions based on their knowledge of the specific exergame systems and activities.

\section{Expert clinician factors}

Communication (facilitators and barriers). Some clinicians mentioned that they had very good communication with the expert clinician and received a lot of feedback. However, another clinician expanded by stating: "I get feedback if they like it, but I don't get feedback throughout to say they're doing really well, they're improving. I haven't heard any, no."

Client safety (facilitators). The presence of the expert clinician in the Exergames Room was viewed by most clinicians as a facilitator as it ensured client supervision. One therapist said: "if there would be nobody there, I'd have to change my intervention to ensure safety."

Practicality (facilitators). Clinicians felt the presence of the expert clinician and of the assistant made the room easier to use as they did not need to worry about setting up the room themselves. "[Translated] ... I find it good that we have an expert clinician to whom we can refer patients and she tells us if they are good candidates or not. Also, I know that she reevaluates to adjust the program for each client". This allowed clinicians to have more time for their own therapy sessions.

Reliability (facilitators). Clinicians reported trusting their clients in the hands of this expert clinician or "champion OT" as one clinician nicknamed her. They expressed complete confidence in this OT's skills and expertise.

\section{Client factors}

Personal factors (facilitators and barriers). In terms of personal factors related to the client, most clinicians indicated motivation as an important facilitator regarding the referral to the Exergames Room. However, personal factors that negatively influenced the use of the Exergames Room included transportation and financial difficulties for out-patients who had to commute to the hospital more often. Also, clinicians reported hesitation towards referring clients who are not technology aware. During interviews, some clinicians attributed this lack of knowledge in technology to be more common in the elderly population. Finally, a period of time and practice was required for clients to adapt to the Exergames Room and to feel confident with the activities.

Relevance of Exergames Room for different levels of function (facilitators). Clinicians found the Exergames Room to be relevant for clients with a wide range of abilities. This was reflected when a clinician found "[translated] [the Exergames Room] to be just as applicable for a low level client in wheelchair as someone who is able to stand on a balance board". Other clinicians reported a similar opinion saying that the room was relevant for those who had minimal movement in their affected limbs and minimal trunk control as well as those who showed good motor function in their affected limbs.

Feedback from clients (facilitators). Clinicians received positive feedback from clients that acted as a facilitator in their decisions to refer new clients. Examples mentioned were: "they appreciated the room", "they are satisfied" and "they like the games".

Functional limitations (barriers). Functional limitations of the clients that served as barriers included fatigue, communication limitations, physical limitations, cognitive limitations, and level of independence. In terms of fatigue, clinicians felt that some clients could not handle the extra therapy due to fatigue and low endurance. One clinician provided an example of a client's pain limiting her referral as she said: "[translated] I wasn't sure...I did not refer her...I did not recommend [the Exergames Room] because she had pain on her right side therefore I did not want to overstimulate her right affected side". Communication barriers were identified in clients with aphasia, which made it difficult to participate in the Exergames Room. Physical barriers included flaccid ULs and very low motor function as clinicians felt that sensorimotor function in these clients would plateau early, limiting their functional gains from the Exergames Room. Cognitive imitations and perceptual difficulties were perceived as barriers by clinicians as they affected participation and how much these clients could benefit from the room. Finally, other clinicians mentioned that they did not feel that VR was appropriate for clients with very low functional level.

\section{Technological level}

\section{VR system}

Games (facilitators and barriers). More than half of the participants reported that the variety of activities positively influenced their referral decision. For instance, they mentioned that the games were functional, provided bilateral tasks, and worked on versatile goals. On the other hand, some clinicians felt that the games failed to challenge clients on their cognitive, social and problem-solving abilities.

Client/clinician perceived ease of use (facilitators). In terms of the VR system, some clinicians perceived it as "user-friendly" since it could benefit clients of all ages, even those who are not familiar with technology.

$V R$ system feedback (facilitators). Clinicians appreciated the feedback of movement seen on screen, as well as the feedback received through in-game scores. A clinician stated: "I think [the games are] a good thing because you can actually increase the level of difficulty and you get a score so there is feedback."

Performance of VR system (barriers). Some clinicians felt that the lack of precision in movement detection and the lack of task specificity were barriers allowing clients to cheat and use compensatory movements. One clinician mentioned that they felt that the motion capture system was unsafe, because the expert clinician could not stand too close to the client without the motion sensor capturing the clinician instead of the client. Finally, one clinician 
stated that "it is virtual so it is not real" thus emphasizing the lack of realism.

\section{Therapeutic benefits}

Additional therapy (facilitators). All participants saw the Exergames Room as an opportunity for clients to exercise outside of their regular therapy sessions. Some therapists expressed that it was a way to increase exposure to activities, complementing their therapy time. This is consistent with current practice recommendations that "an additional therapy session per week will always be good" (clinician 01).

Rehabilitation benefits (facilitators). Various benefits to rehabilitation were identified by clinicians, ranging from physical to spiritual benefits. Physically, exergaming systems were identified as beneficial to stimulating movement of the UL and hand, trunk control and sitting balance. Improving endurance was also identified as a benefit. Finally, one clinician spoke about a personal benefit stating: "I think that it also promotes empowerment, that the clients like it and you're enabling them to do what they like then I think that's very important."

\section{Discussion}

The aim of the study was to identify the facilitators and barriers perceived by clinicians to using an Exergames Room as an adjunctive therapy in a stroke rehabilitation setting. While previous qualitative studies have described clinicians' perspectives on $V R$, only a few of them took place in a stroke rehabilitation setting, where clinicians made use of an exergames program over an extended time period. A previous study involving PTs working with children with acquired brain injury have highlighted the ability of VR to provide multisensory feedback and to increase client motivation, allowing for additional practice repetition [12]. Similarly, our participants reported that the VR systems in the Exergames Room provide real-time visual feedback such as game scores. Additionally, a majority of participants greatly appreciated the interest and motivational values clients gained within the Room. Another major facilitator that emerged from the interviews was how the Exergames Room enabled participants to obtain more practice and increase their sense of empowerment.

According to a previous study, one important barrier to the use of exergames in stroke rehabilitation is the limited number of options to individualize treatment plans through game settings [12]. Conversely, most of our interviewees reported that the variety of available games performed in sitting to standing as well as the flexibility of difficulty settings were relevant for clients with a wide range of function. However, clinicians felt that the existing VR systems were not relevant for those with poor tolerance to activity, difficulty with communication, severe cognitive impairments, lack of interest in videogames, and low potential for motor recovery. While games on the Jintronix and Meditouch platforms can be beneficial to reduce physical impairments, participants suggested further game development incorporating social and cognitive components. Recent efforts have been made to create a conceptual framework for designing feasible game-based cognitive rehabilitation system that benefit rehabilitation processes while also increasing patient motivation [20]. Further development in the area of cognitive rehabilitation should be encouraged as the incidence of impaired cognitive functions can occur in $40-60 \%$ of elderly patients following transient ischemic attacks, minor strokes, and strokes with minimal neurological deficits [21].

Previous findings have suggested several support systems to facilitate the implementation of VR, including educational opportunities to develop clinical reasoning skills for selection and modification of game parameters based on different client needs, as well as the assistance of support personnel to operate the VR systems [12]. Most interviewees expressed that the educational opportunities offered by the institution were insufficient. As a result, clinicians reported a lack of knowledge of the VR systems and the activities offered, as well as how to operate them. This is consistent with past studies that have described the barriers to the implementation of VR in clinical settings as a lack of time and knowledge [9-11]. However, our interviewees agreed with the evidence supporting exergames in stroke rehabilitation and expressed the desire to receive more training to gain a better understanding of the use of VR systems. More importantly, the inclusion of a part-time expert clinician in charge of evaluating newly referred clients, and creating individualized exergaming plans was enough to encourage referrals. While the expert clinician was only present one day per week for $3 \mathrm{~h}$, interviewees indicated that she was practical, reliable, trustworthy and a key asset for the safety of participating clients. When probed to identify potential areas for improvement, participants commented that feedback and communication from the expert clinician and assistant in terms of the clients' progress could be improved. A few clinicians also suggested increasing the availability hours of the expert clinician and rehabilitation assistant to allow for more clients to participate.

At the institutional level, it is important to have a strong support system in place to increase the use of a new technology by therapists [13]. Other than including an expert clinician, the institution can act as a facilitator by providing the appropriate resources, implementing an easy to use referral system, and increasing the availability and accessibility of the room. The institution also plays an important role in facilitating the communication between the therapists and those operating the room, which was seen as a barrier and a factor to improve on. Finally, a step by step guidance process (orientation, insight, acceptance, change, and retention to change) should be taken into consideration when implementing a new technology that therapists must learn to use [13].

\section{Study limitations}

The potential loss of information during translation of interview transcripts from French to English may have limited the interpretation of the direct quotes analysed. Nonetheless, when needed, researchers consulted each other or their supervisor for confirmation. Furthermore, there may be limited transferability as participants were recruited through convenience sampling and the information obtained was circumstance-specific to the hospital setting in which the study took place. Despite the small sample size, saturation was achieved over the course of the interviews as themes were recurring. Further research with a larger sample representing different professionals and rehabilitation settings is needed to fully generalize the findings. The sample might also include other healthcare professionals and program administrators who are concerned with the program. Additionally, the study had a major focus on the Jintronix system as clinicians were more familiar with this system. Therefore, there is limited generalizability of study findings to all VR systems. Another limitation is the lack of comparisons with results from atypical clients. All clinicians recruited had referred clients to the Exergames Room while those who did not refer clients could have added different perspectives to the study. Finally, interviewer bias may have been present due to the structure of the interviews. To limit this bias, pre-written 
questions were pilot-tested and used to prevent the interview from deviating from the intended research questions.

\section{Conclusions}

In conclusion, new information was found about how clinicians (PTs and OTs) perceive the use of an Exergames Room as an adjunctive therapy. Different facilitators and barriers were identified to help improve further implementation of VR and exergaming within stroke rehabilitation settings.

\section{Disclosure statement}

The authors report no conflicts of interest.

\section{ORCID}

Philippe S. Archambault (D) http://orcid.org/0000-0002-8656-4477

\section{References}

[1] Weiss PL, Kizony R, Feintuch U, et al. Virtual reality in neurorehabilitation. Textbook Neural Repair Rehabil. 2006;51:182-197.

[2] Joo LY, Yin TS, Xu D, et al. A feasibility study using interactive commercial off-the-shelf computer gaming in upper limb rehabilitation in patients after stroke. J Rehabil Med. 2010;42:437-441.

[3] Laver KE, George S, Thomas S, et al. Virtual reality for stroke rehabilitation. Stroke. 2015;43:e20-e21.

[4] Saposnik G, Teasell R, Mamdani M, et al. Effectiveness of virtual reality using $\mathrm{Wii}_{\mathrm{ii}}$ gaming technology in stroke rehabilitation a pilot randomized clinical trial and proof of principle. Stroke. 2010;41:1477-1484.

[5] Yavuzer G, Senel A, Atay M, et al. "Playstation eyetoy games" improve upper extremity-related motor functioning in subacute stroke: a randomized controlled clinical trial. Eur J Phys Rehabil Med. 2008;44:237-244.

[6] Lang CE, Lohse KR, Birkenmeier RL. Dose and timing in neurorehabilitation: prescribing motor therapy after stroke. Curr Opin Neurol. 2015;28:549-555.

[7] Lang CE, MacDonald JR, Gnip C. Counting repetitions: an observational study of outpatient therapy for people with hemiparesis post-stroke. J Neurol Phys Ther. 2007;31:3-10.
[8] Traicoff K. Meaningful engagement in activity can enhance upper extremity motor performance more than rote exercise in stroke rehabilitation. Phys Funct CATs. 2008;20.

[9] Levac DE, Miller PA. Integrating virtual reality video games into practice: clinicians' experiences. Physiother Theory Pract. 2013;29:504-512.

[10] Hughes A-M, Burridge JH, Demain SH, et al. Translation of evidence-based assistive technologies into stroke rehabilitation: users' perceptions of the barriers and opportunities. BMC Health Serv Res. 2014;14:1.

[11] Levac D, Espy D, Fox E, et al. "Kinect-ing" with clinicians: a knowledge translation resource to support decision making about video game use in rehabilitation. Phys Ther. 2014;95:426-440.

[12] Levac DE, Galvin J. Facilitating clinical decision-making about the use of virtual reality within paediatric motor rehabilitation: application of a classification framework. Dev Neurorehabil. 2011;14:177-184.

[13] Hochstenbach-Waelen A, Seelen HA. Embracing change: practical and theoretical considerations for successful implementation of technology assisting upper limb training in stroke. J Neuroeng Rehabil. 2012;9:1.

[14] Norman KD, Yvonna SL. Constructivist, interpretivist approaches to human inquiry. Chapter 7. In: Handbook of qualitative research. Thousand Oaks: Sage; 1994. p. 118-137.

[15] Jintronix.com [Internet]. Montreal, Canada: Jintronix; [cited 2018 Feb 23]. Available from: http://www.jintronix.com

[16] Gaming F. HandTutor offers enhanced functional and fine motor rehabilitation of the hand. Fitness Gaming; 2016.

[17] Venkatesh V, Morris MG, Davis GB, et al. User acceptance of information technology: toward a unified view. MIS Quart. 2003;27:425-478.

[18] Sabatier P. Top-down and bottom-up approaches to implementation research: a critical analysis and suggested synthesis. J Public Policy. 1986;6:21-48.

[19] Griffin RW, Moorhead G. Organizational behavior: managing people and organizations. Toronto: Nelson Education; 2011. $640 \mathrm{p}$.

[20] Shapi'i A, Mat Zin NA, Elaklouk AM. A game system for cognitive rehabilitation. BioMed Res Int. 2015;2015:493562.

[21] Vakhnina N, Nikitina LY, Parfenov V, et al. Post-stroke cognitive impairments. Neurosci Behav Phys. 2009;39:719-724.

[22] Davis FD. Perceived usefulness, perceived ease of use, and user acceptance of information technology. MIS quarterly. 1989;13:319-340. 